\title{
The ANTARES project
}

\author{
T. Montaruli ${ }^{\mathrm{a}}$, on behalf of the ANTARES Collaboration ${ }^{\mathrm{b}}$ \\ ${ }^{a}$ Physics Department and I.N.F.N., University of Bari, \\ I-70126 Bari, Via Amendola 173, Italy \\ b The ANTARES Collaboration list can be found at http://antares.in2p3.fr
}

The ANTARES deep-sea neutrino telescope will be located at a depth of $2400 \mathrm{~m}$ in the Mediterranean Sea. Deployment of the detector will commence this Autumn and is expected to be completed by the end of 2004 . With a surface area of the order of $0.1 \mathrm{~km}^{2}$ it will be one of the largest European detectors. The aim of neutrino telescopes is to detect high-energy neutrinos from astrophysical sources whilst also providing information on the early Universe. Successful operation of ANTARES in a deep sea environment constitutes an important milestone towards the ultimate goal of the construction of an underwater neutrino telescope at the cubic-kilometre scale. The sky coverage of astrophysical sources offered by a Mediterranean neutrino telescope is complementary to any similar device at the South Pole. The current status of the project is discussed and the expected performance of the detector is described in the context of the scientific programme of the project which comprises astrophysical studies, dark matter searches and neutrino oscillations.

\section{Introduction}

A promising challenge for exploring the Universe is the detection of high-energy $(\gtrsim 1 \mathrm{GeV})$ neutrinos. Such neutrinos could be produced by powerful cosmic accelerators, for example those in supernova remnants, active galactic nuclei, compact binaries, such as micro-quasars, and those producing gamma-ray bursts. Neutrino astronomy complements high-energy gamma astronomy since the early Universe cannot be probed with high-energy photons due to photon-matter and photon-photon interactions - gamma rays of a few hundred $\mathrm{TeV}$ from the Galactic Centre cannot survive their journey to the Earth. The weakly interacting nature of neutrinos, coupled with the fact that they point to their source of origin without deviation makes them unique 'probes' with which to investigate regions at distances larger than $50 \mathrm{Mpc}$.

The production of neutrinos in cosmic accelerators requires hadronic mechanisms to be active in the source. Whilst most of the observed high energy emissions to date can be explained by electromagnetic processes, recent observations of gamma-rays up to $5 \mathrm{TeV}$ from SNR RXJ1713-
39 by the Cherenkov telescope CANGAROO [1] suggest that electromagnetic mechanisms are inconsistent with the data but $\pi^{0}$ decay models can readily explain the measured energy spectrum. There is, however, some debate on this result 2 .

The technique employed by neutrino telescopes is dictated by the small neutrino cross section and the large background due to atmospheric muons. Natural Cherenkov radiators, such as water or ice, provide a large active volume at reasonable cost and the indirect detection of neutrinos through muons produced in charged current interactions increase the 'target' region by the muon range. Detectors are constructed at large depths where the atmospheric muon flux is significantly reduced compared to that at the surface. Upward-going muons produced by neutrinos having crossed the Earth, are recognised as the products of neutrino interactions in or close to the instrumented region.

The Cherenkov light emitted by charged particles in deep water or ice is detected using an array of photomultiplier tubes (PMTs) which are housed, together with some associated electronic components, in a high pressure-resistant glass sphere known as an optical module $(\mathrm{OM})$. The 
muon direction and energy are measured using the arrival times and amplitudes of the PMT pulses. The detector sensitivity increases with energy due to the increase in the $\nu$ - $\mathrm{N}$ cross section, the longer muon range and the increase in the amount of emitted Cherenkov light through secondary particles.

\section{Status of the ANTARES project}

The ANTARES (Astronomy with a Neutrino Telescope and Abyss environmental RESearch) project [3] started in 1996 and involves physicists, sea science experts and engineers from France, Germany, Italy, Russia, Spain, The Netherlands and the United Kingdom. The ANTARES location $\left(42^{\circ} 50^{\prime} \mathrm{N}, 6^{\circ} 10^{\prime} \mathrm{E}\right)$ coupled with the Earth's rotation gives an annual sky coverage of about $3.5 \pi$ sr. The instantaneous overlap with the AMANDA experiment, located at the South Pole [4], will be approximatively $0.5 \pi$ sr giving an integrated common coverage of $1.5 \pi \mathrm{sr}$. The Galactic Centre, an important potential source of highenergy neutrinos, will be visible for $67 \%$ of the day.

The detector will be installed at $2400 \mathrm{~m}$ depth in the Mediterranean Sea, $37 \mathrm{~km}$ off-shore of La Seyne sur Mer, near Toulon (France). An extensive programme of site evaluation has provided the relevant environmental parameters of the detector site. Surveys have confirmed the suitability of the sea bed for detector deployment and sea currents have been measured, they average $\sim 6 \mathrm{~cm} / \mathrm{s}$ with a maximum observed value of $19 \mathrm{~cm} / \mathrm{s}$. The average loss of light transmission through OM glass spheres due to bio-fouling and sedimentation has been determined by longterm measurements to be only $\sim 2 \%$ at the equator of a glass sphere one year after deployment. Moreover, this loss is a decreasing function of the zenith angle [5]. In order to be sure that the biofouling effect will be negligible for long-term measurements, ANTARES PMTs will be oriented downwards.

Water transparency affects the light detection efficiency and thus determines the natural scale for spacing the OMs. The amount of light scattering predominantly affects track reconstruction and is a factor having a relevant effect on the angular resolution. For blue light the absorption length is $\sim 55 \mathrm{~m}$ while the effective scattering length (the scattering length divided by $1-\langle\cos \theta\rangle$, where $\theta$ is the scattering angle) is above the $100 \mathrm{~m}$ range. At the ANTARES site the light scattering contribution is small compared to Antarctic ice. In fact, $95 \%$ of the photons emitted by a pulsed isotropic LED source at a distance of $24 \mathrm{~m}$ from a PMT are collected within $10 \mathrm{~ns}$ (30 ns) for blue (UV) light.

The detector, illustrated in Fig. 1. consists of a 3-dimensional array of OMs [6] arranged in strings made of mechanically resistent electrooptical cables. They will be anchored at the sea bed and held taut by buoys at the top of the string. At least 10 strings each equipped with 90 OMs arranged in 30 storeys are foreseen. Strings will be separated on the sea bed by approximately $60 \mathrm{~m}$ and storeys will be vertically separated by $12 \mathrm{~m}$. Each storey is equipped with $3 \mathrm{OMs}$ oriented at $45^{\circ}$ to the downward vertical. Readout electronics for each group of $3 \mathrm{OMs}$ are located in a titanium container mounted just above the OMs. The 10" Hamamatsu R7081-20 PMTs in the OMs are sensitive to single photons [6]. The front-end electronics of ANTARES OMs includes the Analogue Ring Sampler (ARS) ASIC [7] which has been developed to process PMT signals and measures the arrival time and charge for single photoelectrons (99\% of the events) and the full pulse shape for larger photoelectron signals. The ARS has been shown to have an intrinsic time resolution of $\sigma=0.3 \mathrm{~ns}$. The overall time resolution, including the PMT transit time spread, is about $\sigma=1.5$ ns. Signals are digitized, then transmitted via optical fibres in cables. The cables from individual strings will be connected (using a submarine) to a junction box at the end of an electro-optical cable which sends signals to a shore station where data are recorded. LED beacons (one per six storeys) will be able to emit precise short pulses of blue light in order to calibrate the detector's timing. An extra string, called the "Instrumentation String", will be devoted to the measurement of environmental parameters and will include, at its base, a laser beacon that will also contribute to the timing cal- 
ibration.

Trigger logic and electronics, track reconstruction and background rejection must take into account the presence of background light. The background rate is seen to consist of two constant components: the rate due to the $\beta$-decay of ${ }^{40} \mathrm{~K}$ present in sea water and a continuum rate that varies on a time scale of several hours due to bioluminescence. The background light from these components produces an almost constant counting rate of about $60 \mathrm{kHz}$ on a 10" PMT. Furthermore, short bursts of bioluminescence have been measured with a rise time of a few milliseconds and a few seconds duration. These bursts induce a $\mathrm{MHz}$ level counting rate in a PMT which leads, on average, to less than $5 \%$ dead time on any individual PMT with negligible correlation between storeys.

The ANTARES collaboration has successfully achieved the following:

- from Nov. 1999 to June 2000, a so-called "demonstrator string" (of a different mechanical design to the current design) was deployed at a depth of $1100 \mathrm{~m}$ and connected to shore with a $37-\mathrm{km}$ electro-optical cable. This string was instrumented with 7 PMTs and more than 500007 -fold coincidences were recorded, zenith angles were reconstructed using times and amplitudes. The shape of the zenith angular distribution of atmospheric muons was reproduced reasonably well, despite there being only a small number of PMTs and a single string [8]. After reconstruction, the data were seen to include a 50\% contribution of multiple muons - as expected from such a shallow site. The demonstrator string allowed the ANTARES relative and absolute positioning to be tested with a system of acoustic rangemeters, compasses and tiltmeters. Relative distances between 2 elements were measured with $5 \mathrm{~cm}$ accuracy and absolute positioning was obtained with $\sim 1 \mathrm{~m}$ accuracy. These values garantee a sufficiently accurate positioning for astrophysics searches.

- A test of OM sphere implosion was per- formed in June 2000 using a mechanical structure consisting of 2 mechanically complete but un-instrumented storeys. One OM was artificially weakened and imploded at a depth of $2600 \mathrm{~m}$. After the implosion, the other spheres and the electronics box in the same storey had also imploded, but the components in the adjacent storey and the mechanical cable remained intact;

- the electro-optical cable to carry electrical power and data between the ANTARES site and shore was successfully deployed in October 2001;

- a mechanical test of a prototype string deployment has been performed in November 2001;

- in April 2002 the industrial production of 900 Optical Modules started.

The planning of the experiment includes the following key dates:

- Autumn 2002: deployment of a prototype string containing 5 storeys (called sector string) and of the instrumentation string;

- December 2002: connection to the junction box and start of data taking;

- June 2003: deployment of first two strings;

- December 2004: operation of the 10 string detector.

\section{Expected performance}

The ANTARES scientific programme is mainly devoted to the detection of neutrinos of astrophysical origin produced in point-like sources or coming from the whole sky as a diffuse flux. The neutrino signal can be detected above the background due to atmospheric neutrinos at energies greater than 10 to $100 \mathrm{TeV}$ as a result of the harder neutrino spectrum expected from cosmic accelerators compared to neutrinos from cosmic ray interactions in the atmosphere. Unfortunately, estimates of neutrino fluxes at large energies are affected by an uncertainty of about 


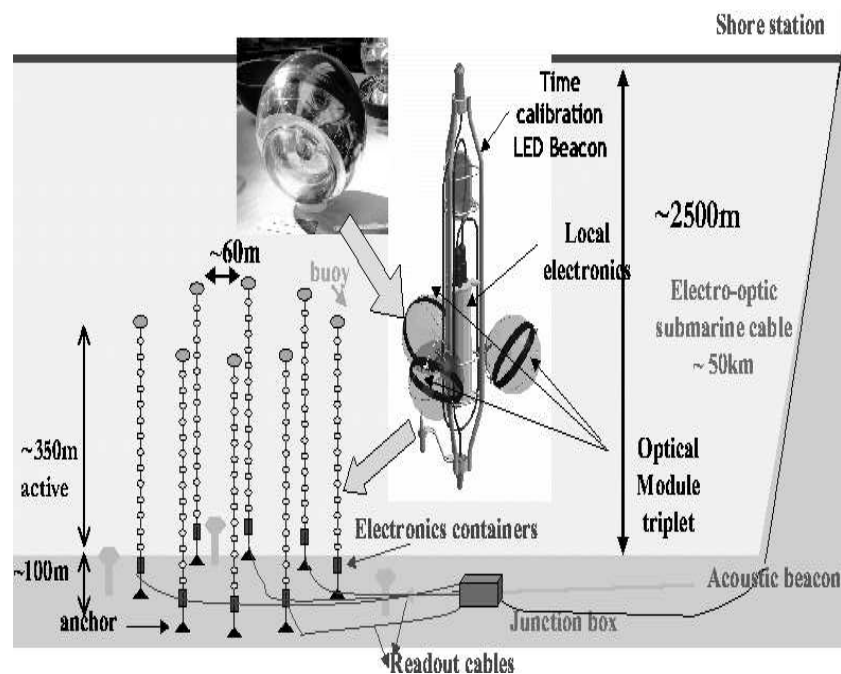

Figure 1. Schematic view of the ANTARES detector showing an OM and some details of a storey enlarged.

2 orders of magnitude on the prompt neutrino contribution coming from charmed meson decays. Since their spectrum is harder than those of the pion/kaon component of the atmospheric neutrino spectrum, prompt neutrino fluxes could constitute a relevant source of background for the cosmic neutrino diffuse flux search, while for point-like and transient sources the knowledge of direction and time will help to suppress this background.

An intensive study to simulate the 10 string detector response to different neutrino fluxes and to reconstruct events has been undertaken. The muon production in the neutrino interaction has been simulated by using deep inelastic scattering with CTEQ3-DIS parton functions [9]. The Earth shadowing effect due to increasing neutrino cross-sections is properly taken into account. Muons are propagated to the detector using MUM code 10 taking account of different media (rock/water). The water properties are simulated including the scattering of photons and a $60 \mathrm{kHz}$ background count rate due to ${ }^{40} \mathrm{~K}$ decays. A simulation of the detector response including the behaviour of the PMT and front-end electronics is also performed.

The important parameters which characterise a neutrino telescope are its effective area (which includes reconstruction and selection efficiencies), the angular resolution and the energy resolution. The muon effective area, a ratio of the selected event rate to the flux of incident muons, depends on the selection criteria used for any specific analysis. For point-like source searches, particularly when a signal should be observed across the entire sky and not necessarily associated to any known object, strict selection criteria are mandatory to keep only well reconstructed events. Thus, good pointing accuracy and a good rejection of the background of atmospheric muons is necessary. Other searches, for instance for known transient sources, such as gamma-ray bursts, which are almost background free on timescales of hundreds of seconds, can be performed by replacing stringent cuts on the quality of the reconstruction by an angular cut around the presumed source. In this case, the effective area calculation is made by requiring that the angle between the direction of the reconstructed muon and the neutrino direction is less than a defined angular cut.

The effective area for the ANTARES detector when using these two approaches is illustrated below. Fig. 2 depicts the effective area of the 10string detector for muon events as a function of neutrino energy assuming an isotropic distribution of neutrinos inducing muons. The solid line is obtained applying strict selections in order to have high-quality reconstructed events (see below for the corresponding angular resolution plot in Fig. (4). The upper dashed curve, which exceeds the geometrical area at high energies, is obtained for reconstructed muon events within an angular error lower than $1^{\circ}$ from the neutrino direction after relaxing quality cuts (e.g. for transient source searches).

Fig. 3 illustrates how the effective area depends on the intrinsic angular resolution for events selected by applying strict reconstruction quality cuts. Together with the curve including all selected events, the curves for a reconstruction error of the muon (angle between the simulated muon and the reconstructed one) less than $1^{\circ}$ and than $0.3^{\circ}$ are shown demonstrating that most of the events are well reconstructed. For a typical $E^{-2}$ cosmic accelerator neutrino spectrum [1] $96 \%$ 


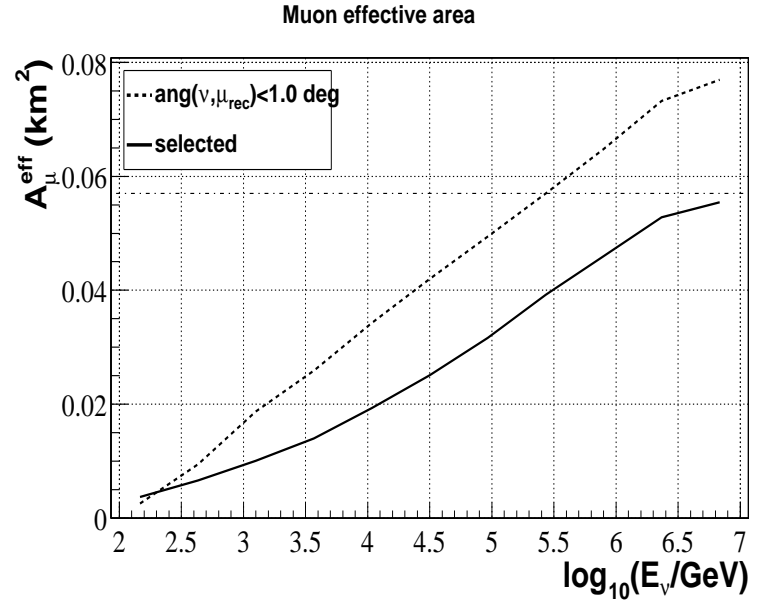

Figure 2. Effective area versus neutrino energy for isotropically simulated events after strict reconstruction quality cuts (solid line) and after relaxing the quality cuts but requiring an angle between the reconstructed muon and the neutrino direction less than $1^{\circ}$ (dashed line). The geometrical instrumented surface is shown as a dasheddotted line.

$(72 \%)$ of the events are reconstructed with an error less than $1^{\circ}\left(0.3^{\circ}\right)$.

The intrinsic angular resolution of the telescope, defined as the median angular separation between the 'true' and the reconstructed muon track, has been estimated. In Fig. 1 the median value of the distribution of the angle between the reconstructed and simulated muon is shown as a solid line, while the dashed line is the median angle between the reconstructed muon and the parent neutrino as a function of the neutrino energy. The reconstruction quality cuts applied to obtain this plot are the same as those used for the solid line in Fig. 2. It should be noted that the improvement of the angular resolution with increasing energy results in an increase of the signal to noise ratio for neutrino astrophysics studies at energies greater than $10 \mathrm{TeV}$. At these energies, the pointing accuracy is only limited by the intrinsic angular resolution with a limiting value of $\sim 0.12^{\circ}$. This estimate includes light scattering effects. Good angular resolution facilitates powerful background rejection when searching for point-like sources. After an optimisation of the

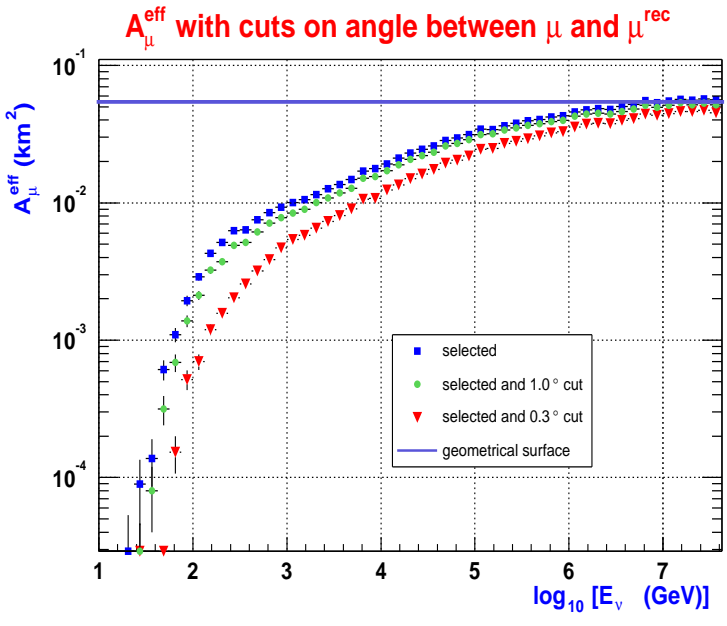

Figure 3. Effective area vs neutrino energy after quality cuts: squares are for all selected events, circles and triangles include the requirement that the reconstruction error is less than $1^{\circ}$ and $0.3^{\circ}$, respectively. The solid line represents the geometrical area.

signal $\left(E^{-2}\right)$ to noise ratio the optimal search cone is seen to have a half-width of $0.7^{\circ}$ around the known source direction or around any measured neutrino event when looking for clusters around it. Furthermore, it is found that the 'optimal' grid in the sky should have $70 \times 70$ bins in right ascension and declination. These results can be compared to those from AMANDA B-10 which report an optimal angular binning of $12^{\circ} \times 12^{\circ} 12$.

\subsection{Sensitivity to point-like sources and to diffuse fluxes}

The effective area in Fig. 2 2 is directly correlated to the properties of the detector. To understand the response to a possible signal, the effective area has to be convoluted with a model-specific neutrino flux. Taking as a reference an $E^{-2}$ signal spectrum, the response curve (differential rate of events versus energy) has a broad maximum around neutrino energies of 10 to $10^{2} \mathrm{TeV}$ and around 3 to $30 \mathrm{TeV}$ of the corresponding muon

${ }^{1}$ While the background is expected to be constant in right ascension, it depends on declination; hence the binning in declination is variable in order to have constant background. 
6

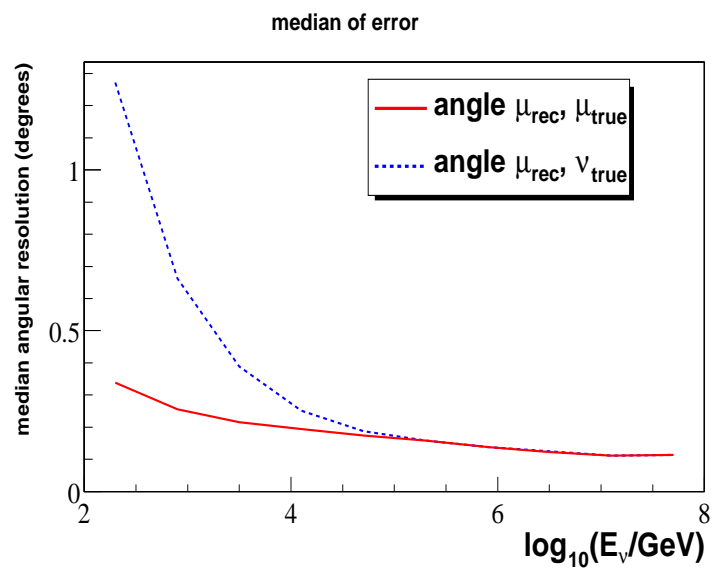

Figure 4. Median angle of the distributions of the angle between the reconstructed muon and the simulated muon (solid line) or the simulated neutrino (dashed line) versus neutrino energy. Below $10 \mathrm{TeV}$ the median angle between the muon and the neutrino is dominated by the kinematics of the interaction, while at larger energies it is limited by the intrinsic angular resolution. The limiting value is about $0.12^{\circ}$.

energies. The maximum of the response curve moves to lower energies for softer spectra. The effective area averaged over an $E^{-2}$ spectrum is of the order of $22000 \mathrm{~m}^{2}$ and decreases for softer spectra. For point-like sources it is also important to consider the dependence of the effective area on declination. This, together with an estimate of the background, allows the upper limits of the muon flux for any given type of source to be calculated. This study is performed considering physical backgrounds, including atmospheric muons and neutrinos. The resulting upper limits in units $\mathrm{cm}^{-2} \mathrm{~s}^{-1}$ of muon flux for 1 year of data taking and for two neutrino models are shown in Fig. 5 where the integration over neutrino energies is made assuming $E_{\text {min }}=10 \mathrm{GeV}$.

To illustrate the expected sensitivity of ANTARES to point-like sources the following example can be considered. Recently a new calculation on neutrino fluxes from galactic microquasars has been published [14 which predicts some promising expected fluxes. In particular, ANTARES expects to measure 6.5 and 4.3 events

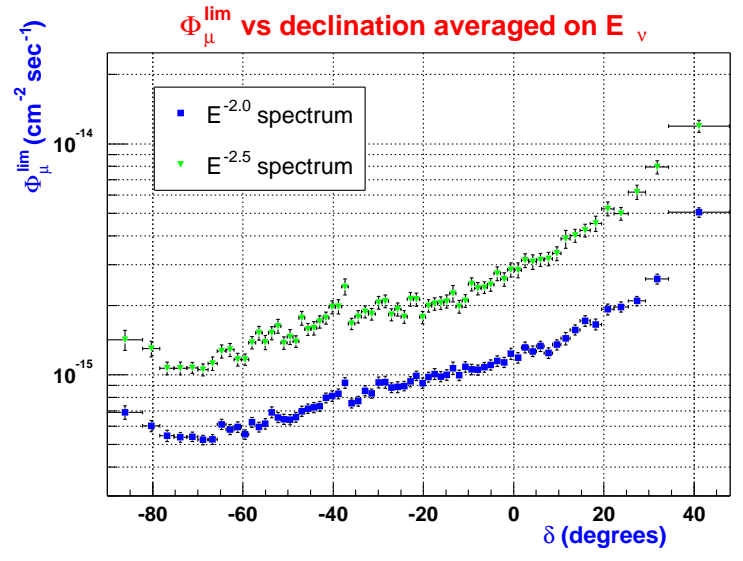

Figure 5. Muon flux upper limits for two power law $\nu$ spectra (upper curve is for spectral index $\gamma=2$ and the lower curve is for $\gamma=2.5$ of a $E^{-\gamma}$ spectrum) after 1 year of data taking. Neutrino energies are integrated between 10 and $10^{7} \mathrm{GeV}$.

per year respectively for GX339-4 and SS433 compared with a background of 0.3 events per year in a $1^{\circ}$ cone.

Diffuse fluxes from astrophysical models are expected to exceed the atmospheric neutrino background at energies above 10 to $100 \mathrm{TeV}$. In Fig. 6 the expected differential event rate versus neutrino energy is shown for atmospheric neutrinos (Bartol flux [15]) and for the Waxman-Bahcall upper limit of $4.5 \times 10^{-8} E^{-2} \mathrm{GeV}^{-1} \mathrm{~cm}^{-2} \mathrm{~s}^{-1}$ $\mathrm{sr}^{-1}$ [16] which is valid for extra-galactic sources transparent to nucleons. The shaded band at high energy is derived from a set of prompt atmospheric neutrino models (references in 17]). Currently the spread between the predictions covers 2 orders of magnitude.

Fig. 6 indicates that the atmospheric neutrino background can be suppressed using the information on the reconstructed energy. Below $100 \mathrm{GeV}$, the neutrino energy is calculated from the range of the muon in the detector (for neutrino oscillation studies), while at energies above $100 \mathrm{GeV}$ an energy estimator based on the PMT charge amplitudes currently allows muon energies to be reconstructed within a factor of 4 at $1 \mathrm{TeV}$ decreasing to a factor of 3 at $10 \mathrm{TeV}$ and reaching the value of 2 in the region 10 to $10^{7} \mathrm{TeV}$. The ANTARES sensitivity to a diffuse neutrino flux 


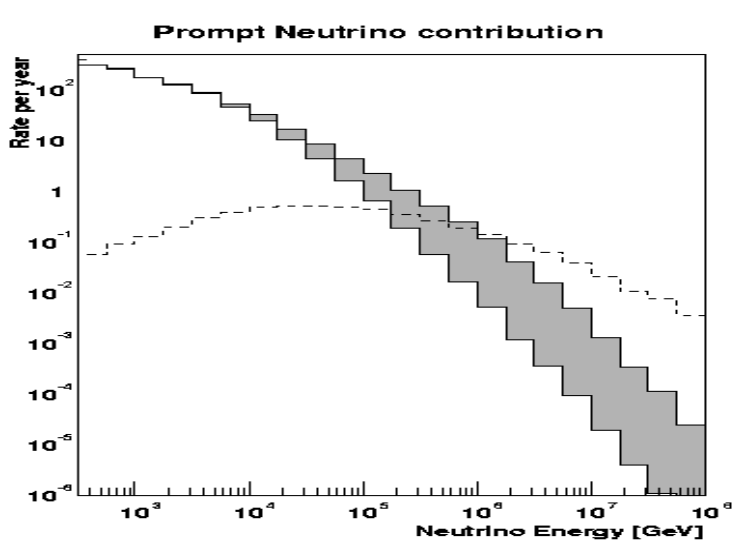

Figure 6. Expected differential rate of events vs $E_{\nu}$ for atmospheric $\nu \mathrm{s}$ [15] and for the upper limit in [16]. The shaded band represents the uncertainty on prompt $\nu$ models (references in [17]).

can thus be estimated by imposing a cut on the reconstructed energy which minimizes the expected background with respect to the signal. The predicted sensitivity is $E^{2} d \Phi / d E=10^{-7} \mathrm{GeV} \mathrm{cm}^{-2}$ $\mathrm{s}^{-1} \mathrm{sr}^{-1}$ above $10^{5} \mathrm{GeV}$, one order of magnitude better than AMANDA B-10 [12] and Baikal 13 but still above the Waxman-Bahcall upper limit [16]. The expected event rates for the atmospheric neutrino background, for a flux equal to the Waxman-Bahcall [16] limit and for the more optimistic upper limit in reference 18] are given in Tab. 1 for reconstructed muon energies greater than $10 \mathrm{TeV}$ and $100 \mathrm{TeV}$. The 'true' event rates for the same threshold on the true muon energy are also quoted as references. The integrated event rates are illustrated in Fig. 7 where solid lines are the expected spectra and dashed lines are the reconstructed ones. The present ANTARES energy reconstruction reproduces harder spectra better than those of atmospheric neutrinos. For harder energy spectrum a small fraction of low energy events with over-estimated energy cannot contaminate significantly high energy events as it is the case for atmospheric neutrinos, which have the steepest energy spectrum. This effect is enhanced by the fact that the energy resolution is a slightly decreasing function of the energy.

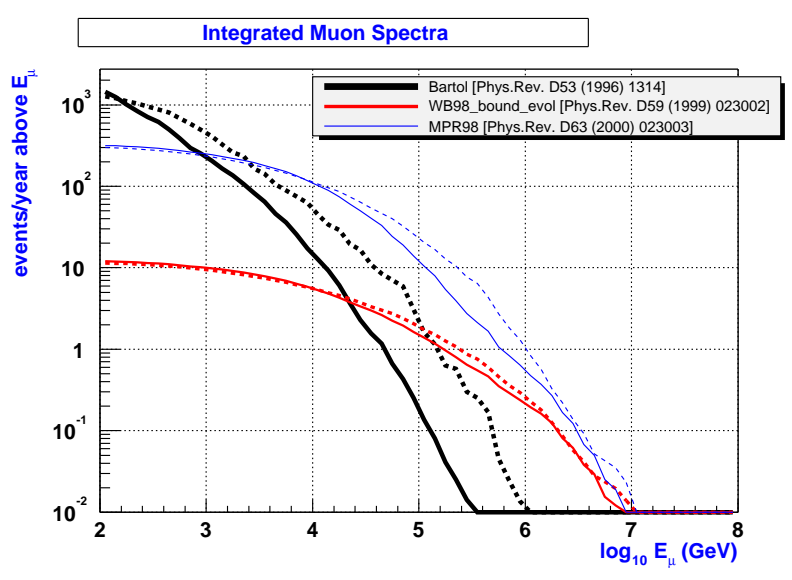

Figure 7. Integrated rate of predicted (solid lines) and reconstructed (dashed lines) muons versus simulated and reconstructed muon energy respectively. The references for the models of atmospheric neutrino fluxes [15] and for upper limits to astrophysical neutrino sources are shown also in the plot (WB98 [16] and MPR98 [18]).

\subsection{Sensitivity to WIMPS}

There is strong evidence in favour of a flat Universe with matter density $\Omega_{\text {matter }} \sim 0.35$. This result, combined with Big Bang nucleosynthesis (baryon density $\Omega_{\text {baryon }} \sim 0.05$ ) and with simulations of structure formation, indicates that most of the dark matter in the Universe could be cold (non-relativistic at decoupling time) and non-baryonic. Weakly Interacting Massive Particles (WIMPs) are considered among the most promising candidates, since a typical weak interaction cross-section would provide the relic density to close the Universe. In a supersymmetric framework, assuming R-parity conservation, a theoretically-favoured candidate is the lightest supersymmetric particle, which in many regions of the SUSY parameter space is the lightest neutralino $\chi$. In minimal supergravity inspired models (mSUGRA), using renormalization group equations and requiring radiative electroweak symmetry breaking, 5 parameters 2 determine the MSSM physics including the neutralino

\footnotetext{
${ }^{2}$ These are: the common gaugino mass $m_{0}$, the common scalar mass $m_{1 / 2}$, a common trilinear coupling $\mathrm{A}$, the higgsino mass parameter $\operatorname{sign} \operatorname{sign}(\mu)$ and the ratio of the Higgs vacuum expectation values $\tan \beta$.
} 


\begin{tabular}{|c|c|c|}
\hline Flux & $\begin{array}{c}\text { events } / \mathrm{yr} \\
E_{\mu}^{\text {reco }}>10(100) \mathrm{TeV}\end{array}$ & $\begin{array}{c}\text { events } / \mathrm{yr} \\
E_{\mu}>10(100) \mathrm{TeV}\end{array}$ \\
\hline Atm. & $44(1.3)$ & $12(0.15)$ \\
$\nu$ s 15 & $5(1.7)$ & $5(1.4)$ \\
Ref. 16. & $106(20)$ & $102(11)$ \\
Ref. 18] & \multicolumn{2}{|c|}{} \\
\hline
\end{tabular}

tribution of the expected signal. For comparison, limits from other experiments are shown.

Table 1

Event rates above muon energy cuts at 10 and 100 $\mathrm{TeV}$ for the Bartol atmospheric neutrino flux [15], for upper limits to extragalactic neutrino fluxes in Ref. [16] and [18]. $E_{\mu}$ and $E_{\mu}^{r e c o}$ correspond to a cut on the simulated energy and reconstructed energy respectively.

sector.

Neutralinos in the galactic halo with typical velocities of few hundreds of kilometres per second could be slowed down sufficiently by scattering inside celestial bodies to become gravitationally trapped in their core. Indirect dark matter searches aim to detect high-energy neutrinos from WIMP annihilation with subsequent heavy quark and gauge boson decays in the core of the Sun, the Earth and the Galactic Centre. Indirect detection is complementary to direct techniques which observe the nuclear recoil from spin-independent WIMP-nucleon scattering in scintillation detectors and in cryogenic phonon Ge and Si detectors. These two techniques have different backgrounds and systematic errors and they have better sensitivity in different regions of parameter space. For axially-coupled neutralinos indirect detection is favoured because the Sun is largely made of hydrogen. In addition, dark matter in the Galactic Centre is not accessible to direct techniques.

Indirect searches aim to detect a statistically significant excess against the background of atmospheric neutrinos pointing back towards the Sun, the Galactic Centre and the nadir respectively. Recent simulation results on ANTARES sensitivity have been presented in Ref. [20]. In Fig. 8 the sensitivity curve (90\% c.l.) for 3 years of data taking as a function of neutralino mass is shown. Models are described in 20,21]. The search cone for neutrino events around the core of the Sun has been optimized considering the angular dis-

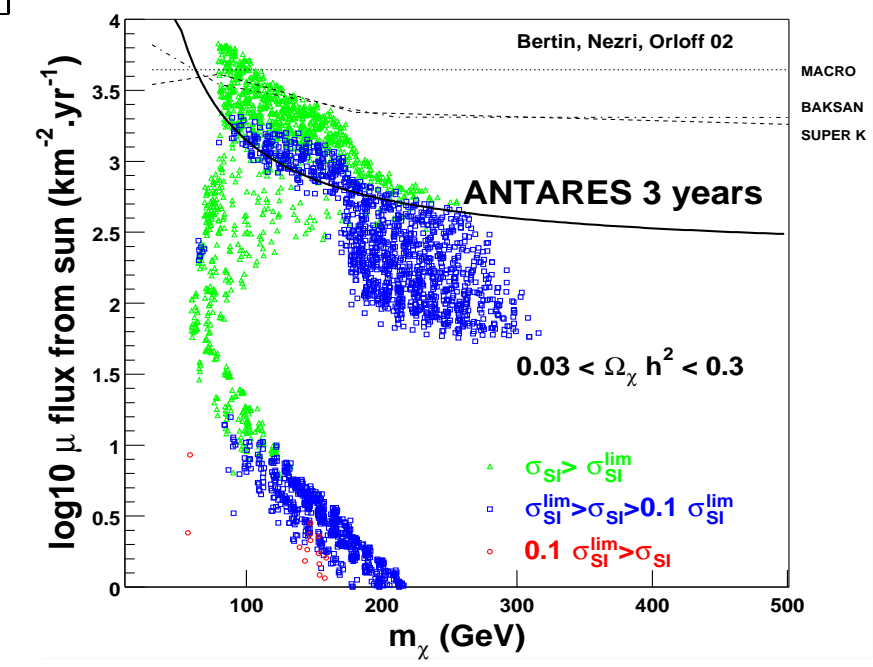

Figure 8. ANTARES (10 strings) 90\% c.l. sensitivity for neutralinos from the Sun for 3 years of data taking as a function of neutralino mass for a hard neutrino spectrum $(\chi \chi \rightarrow W W)$ compared to models described in 20,21. The minimum neutrino energy assumed is $5 \mathrm{GeV}$ for the ANTARES sensitivity curve and for the models. $\sigma_{S I}^{l i m}$ corresponds to the limit on the spinindependent cross-section that can be set after about 3 years of data-taking of Edelweiss II detector [19]. The upper limit is estimated using the Feldman and Cousins unified approach [22]. For comparison, limits from the indirect experiments MACRO [23], Super-Kamiokande [24] and Baksan [25] are superimposed.

\subsection{Atmospheric neutrino oscillations}

Three different neutrino event topologies can be used to study neutrino oscillations: contained events with the vertex and the induced muon inside the implemented region, semi-contained events with the vertex inside the detector but with the muon leaving it and through-going events with the vertex outside the detector. Work is in progress to understand the systematic effects 
and to improve the precision on the oscillation parameter measurement. The measurement of the ratio, $\mathrm{E} / \mathrm{L}$, of the neutrino energy to the baseline length through the Earth for atmospheric neutrinos can provide evidence of neutrino oscillations. The best estimator of this quantity is $E_{\mu} / \cos \theta$, where $E_{\mu}$ is connected to neutrino energy even though the hadronic component takes away part of the energy in the interaction, and $\cos \theta_{\mu}$ is proportional to $\mathrm{L}$, even though at neutrino energies less than about $10 \mathrm{GeV}$ the average angle between the muon and the neutrino is not negligible. The visible muon energy is reconstructed from the muon range for contained events selected by a containment cut based on PMTs along the track that have not been hit; for partiallycontained events an estimated correction is applied. An analysis using the 720 single-string and 2100 multi-string contained and semi-contained events expected in 4 yrs of data taking indicates that the oscillation pattern can be reconstructed in the region allowed by Super-Kamiokande (see Fig. 9).

\section{Conclusions}

The ANTARES neutrino telescope is foreseen to be fully deployed by the end of 2004 . The $\mathrm{R} \& \mathrm{D}$ phase of the project, which started in 1996, has finished. During this phase a detailed assessment of the main requirements for an undersea neutrino telescope was made. The phase culminated in 1999 with the successful operation for several months of a demonstrator string which allowed the reconstruction of atmospheric muons. The project has now entered the construction and deployment phase for a $0.1 \mathrm{~km}^{2}$ scale detector. An electro-optical cable has been successfully deployed and a prototype string and a string dedicated to environmental parameter measurements will be deployed during this Autumn.

Simulations tools are providing relevant information concerning the scientific programme of the experiment, namely neutrino astrophysics, dark matter and neutrino oscillations. The predicted ANTARES sensitivity to fluxes from cosmic accelerators are about an order of magnitude better than results presented to date by other neutrino telescopes.

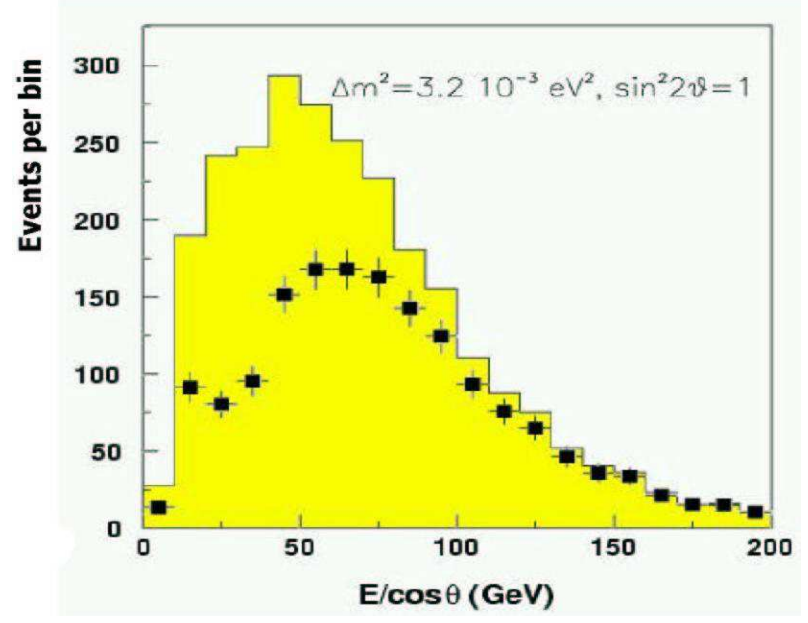

Figure 9. Distribution of $E_{\mu} / \cos \theta$ which would be obtained by ANTARES after 4 years of data taking (data points) assuming $\Delta m^{2}=3.2 \cdot 10^{-3}$ $\mathrm{eV}^{2}$ and maximal mixing. Errors are statistical only. The histogram is obtained assuming no oscillations.

\section{REFERENCES}

1. R. Enomoto et al., Nature 416 (2002) 823.

2. O. Reimer et al., submitted to Astron. Astrophys. and astro-ph/0205256.

3. ANTARES Proposal, http://antares.in2p3.fn

4. D. Ahrens et al., AMANDA Collaboration, astro-ph/0205109.

5. P. Amram et al., ANTARES Collaboration, Sedimentation and fouling of optical surfaces in the ANTARES site, submitted to Astrop. Phys. and astro-ph/0206454.

6. P. Amram et al., ANTARES Collaboration, The ANTARES Optical module, Nucl. Instr. and Methods A484 (2002) 369.

7. F. Feinstein, The Analogue Ring Sampler: a front-end chip for ANTARES, talk given at the $3^{\text {rd }}$ Beaune Conference on New Developments in Photodetection (Beaune 2002), 1721 June 2002, Beaune, France.

8. A. Kouchner, Possibilité d'observation, par le télescope ANTARES, de neu- 
trinos de haute énergie associés aux sursauts gamma et validation des techniques de détection à l'aide d'un prototype, $\mathrm{PhD}$ Thesis, April 2001, available at http://antares.in2p3.fr/Publications/\#thesis; Y. Benhammou, Last results of ANTARES, Frascati Workshop 2001: Multifrequency behaviour of high-energy cosmic sources, 21-26 May 2001, Vulcano, Italy.

9. R. Gandhi, C. Quigg, M.H. Reno and I. Sarcevic, Phys. Rev. D58 (1998) 093009.

10. I.A. Sokalski et al., Phys. Rev. D64 (2001) 074015 .

11. T.K. Gaisser, F. Halzen and T. Stanev, Phys. Rept. 258 (1995) 173.

12. D. Cowen for the AMANDA Collaboration, to appear in Proc. of Neutrino 2002, Munich, 2002, in http://neutrino2002.ph.tum.de

13. G. Domogatsky for the Baikal Collaboration, to appear in Proc. of Neutrino 2002, Munich, 2002, in http://neutrino2002.ph.tum.de

14. C. Distefano et al., astro-ph/0202200 (2002).

15. V. Agrawal, T.K. Gaisser, P. Lipari, T. Stanev, Phys. Rev. D53 (1996) 1314.

16. E. Waxman and J.N. Bahcall, Phys. Rev. D59 (1999) 023002.
17. C.G.S. Costa, Astrop. Phys. 16 (2001) 193.

18. K. Mannheim, R.J. Protheroe, and J.P. Rachen, Phys. Rev. D63 (2000) 023003.

19. G. Gerbier for Edelweiss Collaboration, to appear in Proc. of Neutrino 2002, Munich, 2002, in http://neutrino2002.ph.tum.de

20. L. Thompson for ANTARES Collaboration, to appear in Proc. of DARK2002, Cape Town, South Africa (2002).

21. V. Bertin, E. Nezri and J. Orloff, Neutrino indirect detection of neutralino dark matter in the CMSSM, submitted to European Phys. J. $\mathbf{C}$ and hep-ph/0204135.

22. G.J. Feldman and R.D. Cousins, Phys. Rev. D57 (1998) 3873.

23. T. Montaruli for MACRO Collaboration, in Proc. of School and Workshop on Neutrino Particle Astrophysics, Les Houches (2002).

24. A. Habig for the Super-Kamiokande Collaboration, $27^{\text {th }}$ Int. Cosmic Ray Conf. (ICRC2001), Hamburg, Germany, and hepex/0106024.

25. O. Suvorova, Proc. of Beyond the Desert 99, Ringberg Castle, Tegernsee, Germany, p. 853 and hep-ph/9911415. 